Rev. franç. de linguistique appliquée, 2017, XXII-2 (109-126)

\title{
De la polyfonctionnalité de JE SAIS dans des débats publics et télévisés
}

\author{
Jérôme Jacquin, Université de Lausanne
}

\begin{abstract}
Résumé : L'article examine le marqueur épistémique emblématique de JE SAIS dans un corpus de 10h de débats publics et télévisés. Le défrichage quantitatif révèle une douzaine d'occurrences, ce qui peut sembler faible à la fois au vu de la fréquence, dans le corpus, d'autres expressions épistémiques telles que ON SAIT Ou JE SAIS PAS et en prenant en compte les propriétés interactionnelles et argumentatives des débats dégagées par la littérature existante. Faisant dialoguer la linguistique interactionnelle, la linguistique énonciative et les études sur l'argumentation, cette contribution étudie de manière détaillée le déploiement énonciatif et argumentatif du tour de parole au sein duquel JE SAIS émerge. L'analyse témoigne de la polyfonctionnalité de la ressource, dont les usages ne peuvent se résumer à la revendication affichée d'un état de connaissance.
\end{abstract}

\begin{abstract}
This paper examines the French epistemic marker JE SAIS [I know] in a ten-hour videorecorded corpus of public and TV debates. A statistical survey reveals only a dozen tokens, which appears to be a low frequency both in comparison with other expressions such as ON SAIT [onelyou/we know] or JE SAIS PAS [I don't know] and considering the interactional and argumentative properties of debates, as identified by previous research. Bringing together insights from Interactional Linguistics, Enunciative Linguistics and Argumentation Theory, the paper scrutinizes the enunciative and argumentative unfolding of the turn in which JE SAIS operates. The analysis highlights the polyfunctional dimension of the resource, which cannot be reduced to the self-attribution of a state of knowledge.
\end{abstract}

Mots-clés : Positionnement épistémique, énonciation, interaction, argumentation

Keywords: Epistemic stancetaking, discourse, interaction, argumentation

\section{Introduction}

Le présent article, qui relève d'une analyse multimodale de marqueurs de positionnements épistémiques dans des corpus d'interactions en français, s'intéresse à l'expression emblématique JE SAIS telle qu'elle émerge d'un corpus vidéo-enregistré de $10 \mathrm{~h}$ de débats publics et télévisés. Le corpus révèle la présence de seulement 11 occurrences de JE SAIS, à comparer à la fréquence plus importante d'autres expressions épistémiques, telles que ON SAIT $(n=28)$ et JE SAIS PAS $(n=79)$. Ces 11 JE SAIS sont à certains égards très homogènes : ils introduisent tous des subordonnées complétives (je sais p) et ils apparaissent au milieu de (parfois très) longs tours de parole, ce qui rend délicate leur analyse dans une perspective uniquement interactionniste. Ainsi l'extrait (1) ci-dessous, sur lequel on reviendra et qui est tiré de la première intervention d'une invitée à un débat consacré à la prétendue pollution de la politique par les médias.

Si le JE SAIS et sa complétive des lignes 4-5 émergent certes d'une situation d'interaction, il n'en reste pas moins que l'énoncé ne suscite aucune réaction particulière ; il passe en quelque sorte inaperçu au niveau interactionnel. En d'autres termes et si on s'en tient à l'organisation séquentielle de l'interaction, l'analysabilité de l'action accomplie par JE SAIS pose question, 
dans la mesure où c'est le travail énonciatif et argumentatif opéré par la locutrice qui semble ici au premier plan.

(1) POL-MED / 00:18:00.000

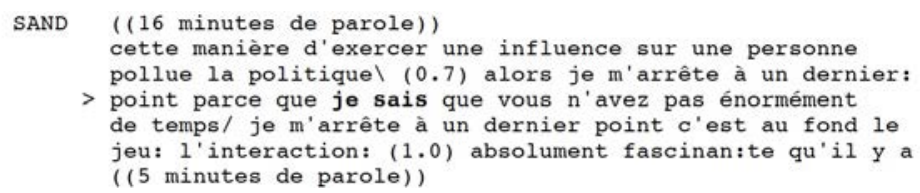

En conséquence, la présente contribution propose une ouverture de la linguistique interactionnelle à des problématiques discursives (principalement énonciatives et argumentatives) comme moyen de rendre justice à la complexité des phénomènes dont relèvent les données considérées. Ce relatif éclectisme théorique et méthodologique sera mobilisé pour explorer attentivement et systématiquement la participation de JE SAIS à l'activité de débat. Ce faisant, la contribution va être amenée à en interroger la valeur plus ou moins épistémique, c'est-à-dire plus ou moins relative au positionnement du locuteur en tant que sujet sachant ou ne sachant pas quelque chose.

La Section 2 brosse un bref état de l'art consacré à l'analyse interactionnelle et argumentative des débats. Elle introduit ainsi la toile de fond de laquelle les 11 JE SAIS émergent ainsi que les différents facteurs pouvant nuancer a priori l'effet de surprise suscité par cette fréquence relativement basse. La Section 3 procède ensuite à un examen approfondi de la collection. Au niveau discursif, on considère la modulation de la prise en charge énonciative des énoncés contenant JE SAIS ainsi que la trajectoire argumentative à laquelle ils participent. Au niveau interactionnel, on évalue la contribution de ces JE SAIS à l'organisation séquentielle et à la dynamique négociative qui caractérise l'activité de débat. Troisièmement et finalement, on rend compte, lorsque les données s'y prêtent (notamment au niveau des prises de vues), de l'environnement sémiotique de ces énoncés pour identifier et commenter la pertinence d'éventuels patrons multimodaux ou 'Gestalts multimodales' (Mondada 2014) associant JE SAIS à une mimo-gestualité particulière. Finalement, la Section 4 synthétise le propos et présente cette contribution comme une occasion de poser les premiers jalons d'une recherche récemment entamée et relevant de l'étude des positionnements épistémiques tels qu'ils participent à l'organisation de différentes activités argumentatives, dont les débats.

\section{Pratique(s) du débat}

\subsection{Dynamiques interactionnelles}

Dans leur examen détaillé des 'systèmes d'alternance des tours de parole' [turn-taking systems], Sacks et al. $(1974,701)$ mentionnent les débats comme activités inter-actionnelles emblématiquement distinctes de la conversation quotidienne, dans la mesure où the size of turns and the ordering of turns in debates, for example, are obviously pre-specified. Si cette remarque ne repose sur aucune analyse systématique, les auteurs étayent plus loin leur intuition :

In debates, the order in which the participants talk is directly related to the character of what they are to say, the parties being characterizable as 'pro' and 'con', and the turns in which they talk as, e.g., 'rebuttal' and 'counter- rebuttal' (ibid., 710).

Le débat relèverait dès lors d'une 'préallocation des types de tours' [turn-type preallocation], dispositif suggérant le caractère marqué, spécialisé ou encore institutionnel de ce type d'interaction. Mais paradoxalement, et à la différence d'autres genres interactionnels 
institutionnels manifestant des contraintes dans les mécanismes de régulation d'accès à la parole, tels que la consultation médicale, les appels d'urgence, les entretiens d'embauche, médiatiques ou de police, ou encore les interactions en classe (Drew \& Heritage 1992), les débats ont fait l'objet d'un nombre très limité d'études se revendiquant de l'analyse conversationnelle d'inspiration ethnométhodologique. De fait, les travaux disponibles se distribuent sur deux axes de recherches complémentaires mais qui se sont rarement croisés : (i) les études consacrées à la gestion de l'attention conjointe et de la participation du public dans des situations à la fois institutionnelles et multi-partites, et (ii) celles qui concernent la configuration et la mise en visibilité plus ou moins spectaculaire de désaccords dans différents genres médiatiques.

(i) Pour ce qui est de la participation du public, Atkinson (1984) décrit différentes techniques discursives - la plupart d'entre elles sont bien connues depuis la rhétorique classique grecque et latine - pour inviter le public à une prise de tour collective et affiliative, sous la forme d'applaudissements ${ }^{1}$. En guise d'exemples, on peut citer les listes de trois (par exemple 'la sécurité', 'la liberté' et 'la prospérité') et les structures contrastives, dont les chiasmes et les oxymores (Heritage \& Greatbatch 1986 ; Relieu \& Brock 1995). Ces analyses ont été aussitôt enrichies, dans les années 1990, de formats d'entretiens radiophoniques ou télévisés qui favorisent la participation active du public, non plus en termes de collectif, mais d'individus qui doivent dès lors s'approprier des occasions de parler (Hutchby 1996; Livingstone \& Lunt 1994). Les études disponibles considèrent la forme et la fonction que prennent ces prises de tour, qui mêlent récits relevant du témoignage et expressions d'opinions personnelles (Hutchby 1999, 2001 ; Thornborrow 1997). Parallèlement, à un niveau moins médiatique que politique, se développent depuis une vingtaine d'années des initiatives de démocratie participative où les citoyens sont directement consultés et encouragés à prendre une part active dans les processus décisionnels. On considère alors la manière, notamment multimodale, dont l'animateur gère l'alternance des locuteurs et donc le droit à la parole (Mondada 2013) ainsi que l'élaboration, progressive et plus ou moins négociée, d'opinions collectivement endossées par les participants (Llewellyn 2005).

(ii) La gestion du désaccord et son influence sur l'organisation de l'interaction constituent l'autre axe de variation au travers duquel se manifestent certaines spécificités des interactions à caractère politico-médiatique. Repartant de Pomerantz (1984), les analyses d'interviews croisées [panel interviews] de Greatbatch $(1992,277)$ montrent que the organization of disagreements between news [interviewees] differs markedly from that of disagreements between speakers in ordinary conversation. Ces désaccords sont en effet systématiquement médiés par un tiers, le journaliste qui anime le débat à destination des auditeurs et téléspectateurs, leur expression n'est pas systématiquement retardée et atténuée et ils ne font pas l'objet d'une orientation systématique et collaborative vers leur résolution. Alors que pour Greatbatch (op. cit., 299) the news interview turn-taking system and its attendant footings supersede the preference features used in ordinary conversation, Kotthoff (1993) conclut plus radicalement à une inversion de la préférence et donc à une préférence pour le désaccord et non l'accord dans ce type de situations. Cette conclusion est plus que nuancée par Doury et Kerbrat-Orecchioni (2011), dans leur analyse des marques d'accord et de désaccord lors d'un débat présidentiel français d'entre-deux-tours. Les auteures observent certes une fréquence relative plus importante des marques de désaccord, ainsi que des marques d'accord portant soit sur des faits ou principes incontestables soit sur des événements très particuliers. Elles soulignent aussi le fait que les marques d'accords sont systématiquement accompagnées d'un étayage, comme le sont les marques de désaccord dans la conversation ordinaire. Toutefois,

\footnotetext{
${ }^{1}$ Pour des analyses de situations désaffiliatives (par exemple les huées), voir Clayman (1993) et McIlvenny (1996).
} 
elles remarquent que c'est aussi le cas pour le désaccord, alors que, s'il s'agissait de l'action préférée dans ce contexte, il n'aurait pas à être justifié. Plus proches de Greatbatch, elles concluent au fait que les débats manifestent une 'orient[ation] vers le désaccord' (op. cit., 83), comme manière d'assurer la mise en visibilité d'options idéologiques divergentes face auxquelles le tiers (notamment les électeurs) doivent faire un choix ${ }^{2}$.

L'orientation vers le désaccord qui caractérise les débats se reflète non seulement au niveau de la gestion locale de la préférence, mais aussi à un niveau plus 'méso' de structuration séquentielle de l'interaction (Jacquin \& Gradoux 2015) : le débat se présente sous la forme d'une séquence étendue relevant d'une confrontation médiée par un tiers et que Burger (2005, 59) segmente de la manière suivante : une phase de 'sollicitation de l'opinion', suivie par une phase de 'confrontation directe de l'opinion' et enfin, en dernier lieu, une phase de 'stabilisation de l'opinion' (voir aussi Hutchby (1996) et Jacquin (2014)). Notons que l'orientation vers le désaccord ne se réduit pas à la phase deux, mais elle est élaborée en amont dans la phase de la sollicitation (cf. note 2) et elle subsiste, en aval, par la sédimentation et non la résolution de la divergence des opinions exprimées.

\subsection{Dimension argumentative et épaisseur énonciative}

Dans les débats, le système d'alternance des locuteurs - qui relève donc d'une préallocation des types de tours médiée par une animatrice ou un animateur - ainsi que l'orientation systématique vers le désaccord et son étayage constituent des conditions particulièrement favorables à l'émergence de (parfois très) longs tours de parole, ou tours multi- UCT (Unités de Construction du Tour) $)^{3}$. En d'autres termes, les prises de parole, attribuées par l'animateur avec ou sans présélection (par exemple en levant la main), sont l'occasion de développements discursifs complexes, où l'énoncé d'opinion est associé à l'anticipation, la contestation ou la concession de l'opinion d'autrui, où certains éléments sont avancés comme prémisses à d'autres, où les différents éléments introduits sont régulièrement ramassés en guise de synthèse et de clôture du tour. Cette discursivité n'est pas l'apanage des experts ou politiciens invités, mais elle intègre un savoir pratique, rhétorique, à disposition du public 'profane' ['lay'], comme le montre bien Hutchby (1999), qui identifie différents patrons typiques de la 'présentation d'opinion' ['opinion presentation'] dans des débats médiatiques.

Cruciale dans les contextes qui nous intéressent ici, l'argumentation a toutefois fait l'objet de très peu d'attention en analyse conversationnelle. On peut citer les travaux de Jackson et Jacobs (Jackson \& Jacobs 1982 ; Jacobs \& Jackson 1980, 1982), qui s'appliquent à une analyse interactionnelle de séquences argumentatives. Néanmoins, les auteurs changeront leur fusil d'épaule quelques années plus tard (Jacobs \& Jackson 1989), pointant les problèmes analytiques posés par l'analyse en paires adjacentes (Jacquin 2014, 182-198) et partant à la recherche de structures plus profondes, au travers d'une analyse en actes de langage (conditions de félicité, intention de communication, force illocutoire). Ils s'alignent ainsi sur le modèle actuellement dominant pour l'analyse de l'argumentation dialogale: le modèle pragma-dialectique d'Amsterdam (Van Eemeren \& Grootendorst 2004). Normative, dans la mesure où il s'agit de mettre en rapport les pratiques argumentatives effectives avec des 'règles de la discussion critique' établies a priori, la pragma-dialectique cherche en outre à 'reconstruire' la structure profonde de l'argumentation, sans accorder beaucoup d'importance à la surface linguistique et interactionnelle. Notamment pour ces raisons, se développe en

\footnotetext{
${ }^{2}$ Cette polarisation s'accomplit aussi au travers des catégories, d'ordre divers, que l'animateur du débat attribue aux invités et qui participent de la configuration du désaccord (Bovet \& Malbois 2011).

3 Voir Selting (2000) pour une introduction à la problématique des tours multi-UCT en analyse conversationnelle, et Jacquin (2014, chap. 2) pour une application au débat et plus généralement aux situations argumentatives.
} 
parallèle, depuis les années 1990, un modèle dialogal de l'argumentation ancré en linguistique (Doury 1997 ; Jacquin 2014 ; Plantin 1996, 2005). Suivant cette perspective,

L'argumentation peut être saisie comme un mode spécifique de traitement verbal du désaccord, qui consiste en la construction de positions solides, c'est-à-dire étayées par un travail textuel de justification et situées par un travail interactionnel et dialogique de positionnement. (Jacquin \& Micheli 2012, 600)

Une telle définition permet d'identifier et d'analyser l'argumentation dans une large diversité de contextes, des plus monologaux et scripturaux aux plus dialogaux et oraux. Plutôt que de voir dans l'argumentation la recherche de la persuasion, qui pose des problèmes importants d'observabilité et de descriptibilité, cette définition situe l'argumentation à l'intersection d'un travail discursif de justification (par l'introduction d'arguments en soutien à une conclusion) et d'un travail de positionnement, non seulement interactionnel (par la séquentialité des prises de parole catégorisables en termes de proposition/opposition), mais aussi dialogique (citation, allusion, prise en compte des autres positions représentées dans le désaccord). On cherche ainsi à saisir l'épaisseur discursive et énonciative de la parole-en-interaction, de manière à rendre compte des phénomènes non réductibles à la dynamique interactionnelle relevant de la négociation du désaccord, notamment la distinction entre la prise en compte d'une position argumentative adverse et la prise en charge de sa propre position. Le désaccord est en effet susceptible d'émerger de l'élaboration énonciative d'un tour multi-UCT, le locuteur pouvant, par un jeu dialogique, citer des positions adverses ou y faire allusion, créer ainsi des effets d'attente et de projection internes, vis-à-vis desquels son argumentation prend la forme d'une contre-argumentation.

Le débat relevant d'une confrontation entre des locuteurs soutenant des positions antagonistes qu'ils justifient par des arguments et qu'ils situent dans le désaccord, on pourrait s'attendre à ce que JE SAIS soit relativement fréquent dans la mesure où l'expression pourrait servir à consolider une position dans le débat par l'introduction d'un argument d'autorité, à positionner un argument par rapport à des éléments avancés par le ou les adversaire(s) 'ne sachant pas', ou encore à servir à un membre du public à légitimer sa prise de parole. Comme on l'a dit, ce n'est pas le cas et il nous faut encore, avant de considérer la collection, dire quelques mots sur JE SAIS et sa dimension épistémique.

\subsection{Dimension épistémique : quelle place pour JE SAIS ?}

Depuis un peu plus d'une dizaine d'années, et notamment les contributions fondatrices de Heritage et Raymond (Heritage \& Raymond 2005 ; Raymond \& Heritage 2006), la dimension épistémique de la parole-en-interaction fait l'objet d'un nombre grandissant d'études ${ }^{4}$. Contrairement aux interactions en classe, aux consultations médicales et à la conversation quotidienne, le débat et plus généralement les pratiques argumentatives n'ont pas reçu d'attention particulière de ce point de vue, que ce soit en français ou en anglais. La présente étude alimente ce courant de réflexion avec la réserve, introduite en début d'article, que toutes les occurrences de JE SAIS considérées ici apparaissent en milieu de tours multi-UCT et contribuent ainsi davantage au développement énonciatif et argumentatif de ces derniers qu'à leur inscription dans l'organisation séquentielle (à distinguer par exemple des analyses de 'I think' chez Kärkkäinen (2003)).

\footnotetext{
${ }^{4}$ Dans une certaine mesure, on trouve déjà cet intérêt pour les ressources langagières et les mécanismes discursifs d'expression de la subjectivité, dont le degré de connaissance, dans le courant de la 'discursive psychology' (Edwards \& Potter 1992).
} 
En admettant le cadre théorique et analytique hybride proposé par la présente contribution, plusieurs remarques apparaissent pertinentes pour éclairer la fréquence relativement faible de JE SAIS dans le corpus.

Au niveau linguistique, il pourrait sembler tout simplement superflu de dire JE SAIS dans la mesure où 'je sais $p$ ' (par exemple 'je sais qu'il fait beau') et ' $p$ ' (par exemple 'il fait beau') sont strictement équivalents dans une perspective logiciste et vériconditionnaliste de la référence (cf. la synthèse de Gosselin (2014) $)^{5}$. Énoncer 'je sais' serait dès lors redondant et donc peu économique dans un échange argumenté de type débat, dont on a dit qu'il repose sur une confrontation dynamique, notamment au niveau de la compétition pour l'accès à la parole. Néanmoins, Gosselin (op. cit.) rappelle, avec Vet (1994), que les fonctions pragmatiques endossées par ' $p$ ' et 'je sais $p$ ' sont très différentes. Il y aurait avec 'je sais $p$ ' une plus-value pragmatique que Gosselin (op. cit., 77) commente en termes de prise en charge énonciative : dans le premier cas ['p'], le locuteur asserte $\mathrm{p}$ [...] ; dans le second ['je sais $p$ '], il présuppose p. En d'autres termes, avec JE SAIS, le locuteur introduit l'information qui suit comme présupposée (le locuteur sait qu'il sait $p$ ), quelle que soit la nouveauté effective de l'information contenue dans $p$ ou la nouveauté qu'il lui octroie par ailleurs. Cette différence invite à intégrer JE SAIS, en général, et les JE SAIS du corpus, en particulier, dans une pragmalinguistique de ce que Gosselin appelle les 'jugements épistémiques' et que je commenterais davantage en termes de 'positionnements épistémiques' pour souligner leur observabilité (inter)actionnelle.

$\mathrm{Au}$ niveau discursif, plus précisément au niveau des dynamiques argumentatives et énonciatives qui caractérisent l'activité de débat telle que commentée supra, la relativement faible fréquence de JE SAIS dans le corpus peut être mise en regard de trois facteurs potentiellement explicatifs.

Premièrement, JE SAIS se heurte à un principe rhétorique voulant que l'ethos de compétence/connaissance consiste en un effet du discours, et non en l'objet d'une autoattribution explicite (Aristote 1991). L'ethos relève en effet de l'image que l'orateur donne plus ou moins stratégiquement et intentionnellement - de lui-même au travers de la manière dont il s'exprime (par exemple ethos de sérieux, de modestie, d'empathie envers son public). En d'autres termes, l'orateur doit se montrer comme sachant, non le revendiquer explicitement (Jacquin \& Micheli 2013). Cette précaution est encore renforcée du fait de l'ethos de modestie, voulant que 'la fierté et l'orgueil [soient] d'invincibles obstacles à la persuasion' (Lamy 1741, 395).

Deuxièmement, et dans la continuité du point précédent, le corpus fait apparaître une fréquence relative bien plus importante de la forme ON SAIT $(n=28)$. Il est dès lors envisageable que le caractère égocentré de JE SAIS, pêchant non seulement par manque de modestie, mais peut-être aussi par manque de validité (comparer 'je sais $p$ ' avec 'telle ou telle étude montre $p$ '), soit évité au profit de l'usage de 'on', dont la plasticité référentielle est bien documentée (Jacquin 2017a ; Jonasson 2006 ; Rabatel 2001).

Troisièmement et finalement, les études consacrées à l'usage de JE SAIS PAS dans l'interaction verbale montrent que l'expression peut certes nier ou infirmer un état de connaissance, mais qu'elle peut également, selon sa position dans le tour de parole, servir à annoncer un tour de parole déviant de la trajectoire séquentielle projetée par le tour de parole précédent ou à sortir du tour de parole et de la séquence en cours (Pekarek Doehler 2016). Il pourrait en aller de même avec la forme positive JE SAIS, qui ne constituerait donc pas

\footnotetext{
${ }^{5}$ Rappelons que les 11 occurrences de JE SAIS introduisent des complétives, ce qui justifie de les analyser sous la forme 'je sais $p$ '.
} 
systématiquement, ou pas seulement, une ressource de revendication d'un état de connaissance et pourrait servir d'autres fonctions pragmatiques.

\section{JE SAIS comme marqueur de positionnement épistémique dans les débats ?}

Le corpus considéré est composé de 8 débats publics vidéo-enregistrés à l'occasion d'une recherche doctorale (Jacquin (2014) pour la version publiée) et de 2 débats télévisés. Les débats publics ont été organisés entre 2007 et 2009 par des associations d'étudiants de l'Université de Lausanne sur des sujets de société ou des initiatives politiques diverses (écologie, financement des études, relation entre études et emploi, salaire minimum, etc.). Les débats télévisés sont des débats Infrarouge diffusés par la Radio-Télévision Suisse Romande. Ceux-ci ont été sélectionnés à des fins de comparaison avec les débats publics, notamment les deux qui font intervenir les mêmes invités, qui ont eu lieu à la même époque et qui concernent des sujets identiques : l'initiative visant à limiter les possibilités de recours des associations écologistes et celle visant à interdire l'immatriculation de véhicules jugés trop polluants et trop dangereux (initiative dite anti-4x4).

\subsection{Aperçu des JE SAIS en comparaison d'autres expressions épistémiques}

Avant d'étudier plus en détail l'émergence des 11 JE SAIS dans leur environnement énonciatif, interactionnel et multimodal, j'opère ici un défrichage quantitatif visant à les situer vis-à-vis de JE SAIS PAS, de ON SAIT et de ON SAIT PAS ${ }^{6}$. Le Tableau 1 présente ainsi pour chaque forme et chaque position (Début, Milieu, Fin ou Totalité du tour de parole) le nombre d'occurrences relativement au type de complémentation syntaxique (absence de complément, compléments adverbiaux, pronominaux, nominaux ou propositionnels).

Tableau 1. Position et complémentation des JE SAIS en comparaison d'autres expressions.

\begin{tabular}{|c|c|c|c|c|c|c|c|c|c|c|c|c|c|c|c|c|c|}
\hline \multirow{3}{*}{\multicolumn{2}{|c|}{$\begin{array}{c}\text { FORME } \\
\text { POSITION }\end{array}$}} & \multicolumn{8}{|c|}{ POSITIVE } & \multicolumn{8}{|c|}{ NEGATIVE } \\
\hline & & \multicolumn{4}{|c|}{ JE SAIS } & \multicolumn{4}{|c|}{ ON SAIT } & \multicolumn{4}{|c|}{ JE SAIS PAS } & \multicolumn{4}{|c|}{ ON SAIT PAS } \\
\hline & & D & $\mathbf{M}$ & $\mathbf{F}$ & $\mathbf{T}$ & D & $\mathbf{M}$ & $\mathbf{F}$ & $\mathbf{T}$ & D & $\mathbf{M}$ & $\mathbf{F}$ & $\mathbf{T}$ & D & M & $\mathbf{F}$ & $\mathbf{T}$ \\
\hline \multirow{5}{*}{$\frac{\sum_{0.4}^{\frac{1}{2}}}{\frac{1}{2}}$} & e complément & & & & & & & & & 1 & 25 & 3 & & 1 & 1 & & \\
\hline & adverbial & & 1 & & & & & & & & 1 & & & & 1 & & \\
\hline & pronominal & & & & & & 4 & & 2 & & 14 & 1 & & & 2 & & \\
\hline & nominal & & & & & & 1 & & & & 3 & & & & & & \\
\hline & propositionnel & & 10 & & & 2 & 17 & 1 & 1 & 2 & 26 & 3 & & & 5 & & \\
\hline \multirow{2}{*}{\multicolumn{2}{|c|}{$\begin{array}{l}\text { TOTAL } \\
\text { TOTAL }\end{array}$}} & 0 & 11 & 0 & 0 & 2 & 22 & 1 & 3 & 3 & 69 & 7 & 0 & 1 & 9 & 0 & 0 \\
\hline & & \multicolumn{4}{|c|}{11} & \multicolumn{4}{|c|}{28} & \multicolumn{4}{|c|}{79} & \multicolumn{4}{|c|}{10} \\
\hline
\end{tabular}

Le tableau fait apparaitre le nombre sept fois plus important de JE SAIS PAS (n=79) vis-à-vis de JE SAIS $(n=11)$, mais aussi la fréquence plus importante des ON SAIT $(n=28)$ par rapport à ON SAIT PAS $(n=10)$. La comparaison de JE SAIS et ON SAIT va dans le sens du point soulevé dans la section précédente (§ 2.3.), à savoir que la plasticité indéfinie de ON SAIT parait préférée au

\footnotetext{
${ }^{6}$ Il est à noter que NOUS (NE) SAVONS (PAS), bien qu'incluant nécessairement JE, a été écarté pour des raisons de représentativité et de lisibilité : il n'y a qu'une seule occurrence, sous la forme négative. En outre, derrière les formes négatives JE SAIS PAS ou ON SAIT PAS ont été comptabilisés respectivement 3 je ne sais, 6 j'en sais rien et 5 je sais plus ainsi que 1 on ne sait, 2 on n'en sait rien et 1 on sait jamais.Le corpus a été intégralement transcrit dans ELAN (<https://tla.mpi.nl/tools/tla-tools/elan〉, consulté le 8 juin 2017), qui autorise des recherches non simplement simples, mais aussi par expressions régulières, permettant ainsi de faire fi des signes diacritiques et d'attraper toutes les occurrences morpho-phonétiques de l'expression considérée.
} 
caractère tranchant du JE SAIS. En résumé, le tableau montre une tendance à l'utilisation de ON SAIT pour la forme positive, JE SAIS PAS pour la forme négative ${ }^{7}$.

On constate également l'homogénéité de la collection des JE SAIS en termes de position séquentielle et de régime syntaxique, ce qui contraste avec la répartition des autres expressions. Au niveau syntaxique, les JE SAIS se présentent sous la forme 'je sais $p$ ', dans la mesure où la seule occurrence régissant un adverbe et non une proposition intervient dans une concession ('je sais bien') portant indirectement sur une proposition. Même si les constructions propositionnelles sont très bien représentées pour les autres expressions, cellesci manifestent davantage de diversité. On constate environ $20 \%$ de constructions pronominales dans le cas de JE SAIS PAS $(n=15 / 79)$, de ON SAIT $(n=6 / 28)$ et ON SAIT PAS $(n=2 / 10)$, et, de manière relativement spectaculaire, un nombre très important de construction sans complément dans le cas de JE SAIS PAS $(\mathrm{n}=29 / 79)^{8}$.

L'homogénéité des JE SAIS se retrouve au niveau de la position séquentielle. Tous les JE SAIS interviennent en position médiane, position qui est également, et largement, la plus représentée pour les autres expressions. En guise de comparaison, Pekarek Doehler (2016), dans son corpus de $20 \mathrm{~h}$ de focus group discussions, relève $55 \%$ de JE SAIS PAS en position médiane $(n=47)$ lorsque JE SAIS PAS est utilisé en formule autonome (c'est-à-dire sans complément syntaxique). Cette proportion est ici de $87 \%(n=25 / 29)$. Ceci tend à confirmer que les débats analysés ici exploitent ces différentes expressions épistémiques comme stratégies de développement argumentatif et non d'entrée ou de sortie de longs tours de parole multi-UCT. Comme commenté dans l'introduction, les implications théoriques et analytiques de la position médiane des expressions épistémiques ne doivent pas être sous-estimées. Pekarek Doehler (op. cit., 161) conclut en effet son analyse des JE SAIS PAS intervenant en milieu de tour de la manière suivante : as opposed to turn-initial and turn-final uses, [...] the mid-turn [JE SAIS PAS] do not operate at the level of sequence organization nor, more generally, sequential organization. En d'autres termes, la catégorisation des JE SAIS PAS en milieu de tour ne peut reposer uniquement sur une analyse de l'organisation séquentielle. Comme on va le voir, il en va de même avec la complexité énonciative et argumentative dont peuvent relever les tours multi- UCT au sein desquels les JE SAIS émergent.

\subsection{Analyse détaillée de la collection}

La collection des 11 JE SAIS peut donner l'apparence d'un éclatement au niveau tant des contextes énonciatifs et argumentatifs d'apparition que des fonctions discursives et interactionnelles endossées. De manière à organiser l'analyse, $\mathrm{j}$ 'ai retenu trois questions générales et autant de plans, qui n'ont de valeur qu'heuristique : (i) Plan argumentatif : quelle est la portée ou orientation argumentative du JE SAIS ? (ii) Plan interactionnel : l'énoncé contenant JE SAIS et sa complétive est-il pris dans la dynamique interactionnelle, c'est-à-dire constitue-t-il en tant que tel une réaction au tour précédent ou fait-il l'objet d'une réaction dans le tour suivant ? (iii) Plan informationnel : l'information introduite par JE SAIS est-elle

\footnotetext{
${ }^{7}$ Un examen plus détaillé de l'asymétrie entre les 11 occurrences de JE SAIS et les 79 occurrences de JE SAIS PAS manifeste des différences notables entre débats publics et débats télévisés. Alors que les débats télévisés font intervenir autant de JE SAIS que de JE SAIS PAS (4, avec une fréquence d'une occurrence par 30 minutes de débat), il y a 7 fois plus de JE SAIS PAS (75, 1 occurrence par 10 minutes de débat) que de JE SAIS (7, 1 occurrence par $85 \mathrm{~min}$ ) dans les débats publics. Ceci mérite une investigation approfondie, qui sera menée dans un stade ultérieur de la présente recherche.

${ }^{8}$ En ne retenant que les constructions faisant intervenir des propositions subordonnées, les JE SAIS PAS $(n=31)$ restent bien plus fréquents que les JE SAIS $(n=10)$.
} 
présentée comme déjà connue et partagée par l'interlocuteur ? ${ }^{9}$ Le Tableau 2 présente la collection des 11 extraits selon ces trois critères. La numérotation et la présentation s'expliquent par les choix opérés pour la suite de l'analyse, à l'occasion de laquelle, pour une raison de place, seule une sous-collection, grisée dans le tableau, sera étudiée et citée en détail, tandis qu'il sera fait ponctuellement référence, en note, aux extraits proches - par exemple les extraits (1b) et (1c) vis-à-vis de l'extrait (1). Les extraits grisés ont été sélectionnés pour la double raison qu'ils ont été jugés représentatifs des dynamiques représentées et qu'ils rendaient possible une analyse multimodale.

Tableau 2. Collection des extraits contenant JE SAIS.

\begin{tabular}{|c|c|c|c|}
\hline \multirow{2}{*}{ Extrait } & \multicolumn{3}{|c|}{ Plans } \\
\cline { 2 - 4 } & (i) Argumentation & (ii) Interaction & (iii) Information \\
\hline I & Planification & $\neq$ interactionnel & inf. déjà connue \\
\hline Ib & Planification & $\neq$ interactionnel & inf. déjà connue \\
\hline Ic & Planification & $\neq$ interactionnel & inf. déjà connue \\
\hline 2 & Planification & $\neq$ interactionnel & inf. déjà connue \\
\hline 3 & Concession & $=$ interactionnel & inf. déjà connue \\
\hline 3 b & Concession & $=$ interactionnel & inf. déjà connue \\
\hline 4 & Témoignage & $?$ interactionnel & inf. nouvelle \\
\hline 5 & Témoignage & $=$ interactionnel & inf. nouvelle \\
\hline 5 b & Témoignage & $\neq$ interactionnel & inf. nouvelle \\
\hline 6 & Expertise & $=$ interactionnel & inf. nouvelle \\
\hline 6b & Expertise & $=$ interactionnel & inf. nouvelle \\
\hline
\end{tabular}

L'analyse de la collection suit l'ordre du tableau, en considérant les extraits où JE SAIS porte sur la planification du dire ( $\$ 3.2 .1$.), ceux relevant d'une concession (\$ 3.2.2.) et finalement les JE SAIS relevant d'un témoignage ( $\$ 3.2 .3$ ) puis d'une expertise (\$ 3.2.4.), les deux derniers fonctionnant dès lors, comme on le verra, comme marqueurs de position épistémique forte, voire dominante. En d'autres termes, on trouve dans cette collection aussi bien des JE SAIS du type 'je sais/savais déjà cela' et des JE SAIS valant pour 'tout le monde, dont moi, le sait' que des JE SAIS qu' on peut paraphraser par 'moi, je sais'.

\subsubsection{Planification : contextualisation et justification du dire}

Dans l'extrait (1), déjà mentionné en introduction et tiré d'un débat portant sur la prétendue pollution de la politique par les médias, le JE SAIS de la ligne 4 intervient aux trois quarts de l'intervention introductive de Suzette Sandoz (SAND), d'une vingtaine de minutes.

Le JE SAIS contribue ici à la transition entre un développement discursif auquel il est mis un terme et un suivant, annoncé comme le dernier ('je m'arrête à un dernier point'). La complétive introduite par JE SAIS - 'je sais que vous n'avez pas énormément de temps' - porte sur la disponibilité des allocutaires (présence de l'indexical 'vous' et d'un geste de pointage paume ouverte, image 2). Il s'agit donc moins ici d'introduire une information nouvelle que de justifier ('parce que je sais') une planification discursive de l'événement compte tenu de ses conditions temporelles.

\footnotetext{
${ }^{9}$ Rappelons que dans 'je sais $p$ ', la réalité de $p$ est toujours présupposée. Cette présupposition se joue au niveau linguistique et ne doit pas être confondue avec le traitement discursif qui en est fait : les locuteurs peuvent tout à fait introduire du contenu en le présentant comme information nouvelle (c'est-à-dire non partagée) tout en jouant sur son caractère présupposément accepté.
} 
(1) POL-MED / 00:18:00.000

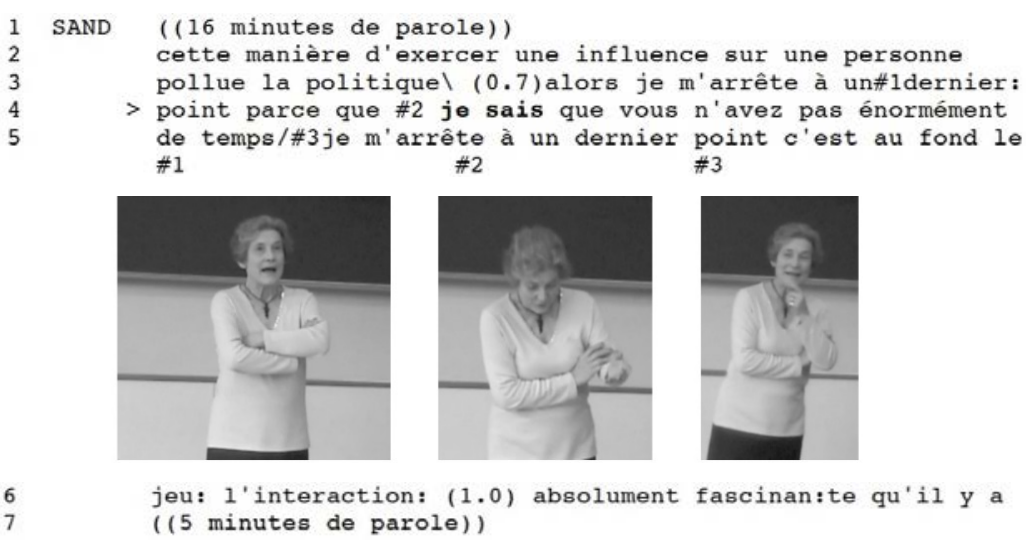

Les JE SAIS des extraits $(1 \mathrm{~b})$ et $(1 \mathrm{c})^{10}$, tirés d'un même débat public consacré à la relation entre études supérieures et marché de l'emploi, fonctionnent de manière analogue. Sur le mode d'un 'je sais/savais déjà', l'animateur utilise JE SAIS pour introduire un account visant à expliquer - on retrouve 'parce que' dans l'extrait (1b) - la trajectoire donnée au tour de parole. Plus précisément, il s'agit pour l'animateur de nier la pertinence actuelle d'une question qui aurait toutefois pu être posée de manière légitime. En d'autres termes, ces 'informations d'arrière-plan' ['background information'] (Clayman \& Heritage 2002, 60) permettent à l'animateur d'écarter de l'agenda une question argumentative, celle-ci faisant l'objet d'un accord complet dans l'extrait (1b) ou d'un désaccord complet dans l'extrait (1c), et d'assurer la transition vers la question retenue, qui sera le focus de la dynamique interactionnelle faisant alterner les phases de proposition et d'opposition (pour une analyse approfondie de l'extrait (1c) et de sa suite, voir Jacquin (2014, 182-198)).

$\mathrm{Si}$ on retourne maintenant à l'extrait (1), on constate, au niveau multimodal, que l'énonciation de JE SAIS et de sa complétive s'accompagne d'un changement ponctuel de direction du regard, du public vers les notes disposées sur la table (images 1-3). Ceci participe du détachement syntaxique de l'incise 'parce que je sais que vous n'avez pas énormément de temps', dont la clôture est signalée par une répétition en aval de la proposition principale ('je m'arrête à un dernier point'). On retrouvera cette délimitation multimodale du segment contenant JE SAIS dans d'autres extraits analysés infra.

L'extrait (2) est tiré d'un débat télévisé consacré à une initiative déposée par les Jeunes Verts suisses et visant à interdire les véhicules jugés trop polluants et trop dangereux. L'animateur vient de clore son introduction et donne la parole à Charles Poncet (PONC), opposé à cette interdiction.

\footnotetext{
${ }^{10}$ Extrait (1b) : 'je vais pas vous demander s'il faut augmenter le financement de l'université ou pas parce que je sais que dans cette salle en tout cas y a pas grand monde qui dira le contraire'. Extrait (1c) : 'Le financement privé [...] alors on parle toujours du modèle américain, ch'sais que ça sert à rien parce que tout de suite ça suscite des éléments qui vont hérisser tout le monde, mais [...] est-ce que le modèle de [l'école cantonale d'art de Lausanne] [...] n'est pas un modèle qu'on devrait un petit peu plus développer'.
} 
(2) RTS.VEH-POL / 00:00:14.000

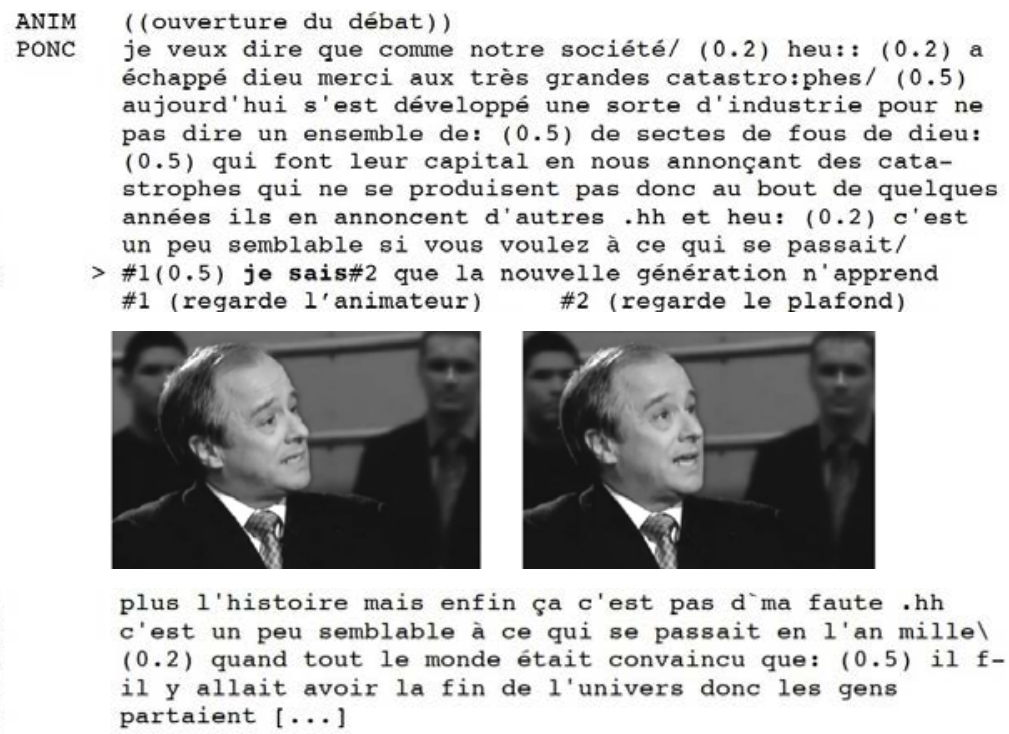

plus l'histoire mais enfin ça c'est pas d'ma faute. hh c'est un peu semblable à ce qui se passait en l'an millel $(0.2)$ quand tout le monde était convaincu que: $(0.5)$ il $\mathrm{f}-$ il y allait avoir la $\mathrm{f}$ in de l'univers donc les gens partaient $[\cdots]$

On retrouve ici un énoncé émergeant hors de la dynamique négociative, sous la forme d'une incise détachée par le regard qui monte ponctuellement au ciel (images 1-2) et par répétition dans son contexte droit de l'énoncé produit à sa gauche ('c'est un peu semblable à ce qui se passait', lignes 9-10). Le contenu énoncé dans la complétive ('la nouvelle génération n'apprend plus l'histoire') est par ailleurs énoncé comme une information déjà connue de tous et donc de l'auditoire (mode du 'tout le monde, dont moi, le sait'). S'il ne concerne pas les conditions temporelles de l'événement, il se présente comme un simple rappel, bien entendu provocateur, de certaines dispositions cognitives, d'une partie de son auditoire, dont les initiants du projet de loi (les Jeunes Verts suisses).

\subsubsection{Concession : intégration du dire de l'autre}

À la différence des cas considérés supra, les JE SAIS qui suivent intègrent des concessions servant d'appui à la contre-argumentation.

L'extrait (3) est tiré - comme l'extrait (1) - du débat sur la prétendue pollution de la politique par les médias. Un désaccord est survenu entre la conférencière et l'animateur, qui insiste pour voir dans les médias un 'pouvoir', ce que conteste la conférencière.

En 3, l'animateur prend ses distances ('c'est pas mon cas') par rapport à la définition du terme 'pouvoir' introduite par SAND en 1-2. SAND reprend alors son tour et interrompt la construction à présentatif 'c'est un groupe de' (ligne 4) pour réagir à la correction de l'animateur, en la concédant ('je sais bien'). S'il s'agit du seul JE SAIS à construction adverbiale du corpus, l'énoncé joue sur l'ellipse d'une complétive de type 'je sais bien [que ce n'est pas votre cas] $]^{11}$. La concession est renforcée par le 'mais' qui suit et qui sert à la fois à concéder l'énoncé à gauche du connecteur et à introduire la contre-argumentation.

${ }^{11}$ La complétive est par contre bien présente dans l'extrait (3b), tiré d'un débat public sur le droit de recours des associations écologistes. Un invité favorable à une limitation de ce droit répond à un membre du public ayant cité l'exemple de Leysin, village qui a bénéficié du dialogue avec les associations pour 
(3) POL-MED / 00:30:45.000

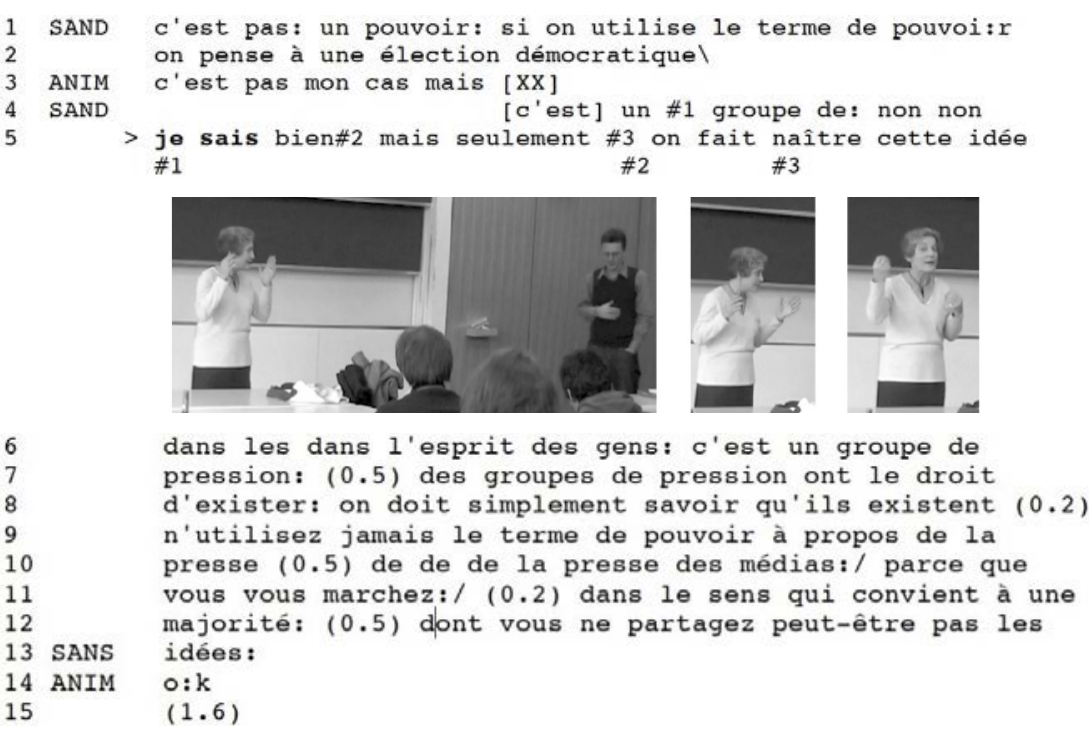

Au niveau multimodal, le geste de cadrage accompagnant la définition à présentatif ('c'est un groupe', image 1, voir Calbris $(2003,85-89)$ ) est interrompu en même temps que la construction syntaxique, pour donner lieu à un geste d'ouverture des mains lors du JE SAIS concessif (image 2). On retrouvera à l'occasion de l'extrait (5) un tel geste d'ouverture figurant l'intégration ou la livraison d'informations dans une opération d' 'explicitation' ( $o p$. cit. 94).

Dans les extraits (3) et (3b), l'information introduite par JE SAIS est concédée, et donc admise ponctuellement sur le mode du 'je sais/savais déjà cela' avant l'introduction d'un élément problématisant qui renverse la trajectoire argumentative. Les cas analysés ci-après diffèrent de ce point de vue : le JE SAIS sera l'introducteur d'une information (plus ou moins) nouvelle sur laquelle l'argumentation prendra prise.

\subsubsection{Témoignage : ouï-dire et expérience personnelle}

L'extrait (4) est tiré du débat public sur la relation entre études supérieures et marché de l'emploi. Il s'agit de la première intervention d'un des dix invités, Jean-Marc Rapp (RAPP).

À la ligne 5, le JE SAIS sert la prise en compte d'une position argumentative en circulation dans l'espace public ('quelques-uns parlent d'une augmentation des taxes', ligne 6) et utilisée comme point d'appui pour l'élaboration de la contre-argumentation ('j'y suis absolument opposé', lignes 6-7).

améliorer un projet de développement : '[...] maintenant vous prenez le cas de peut-être ça s'est très bien passé à Leysin, en l'occurrence je sais que ça s'est très bien passé. Est-ce que ça veut dire que les associations aient le droit de recours? Les discussions vont avoir lieu de toute façon'. L'invité concède l'exemple en l'introduisant dans la complétive de JE SAIS ('je sais que ça s'est très bien passé'), signifiant ainsi le caractère présupposément admis de l'information ('je sais/savais déjà cela'). 
(4) ETU-EMP / 00:16:50.000

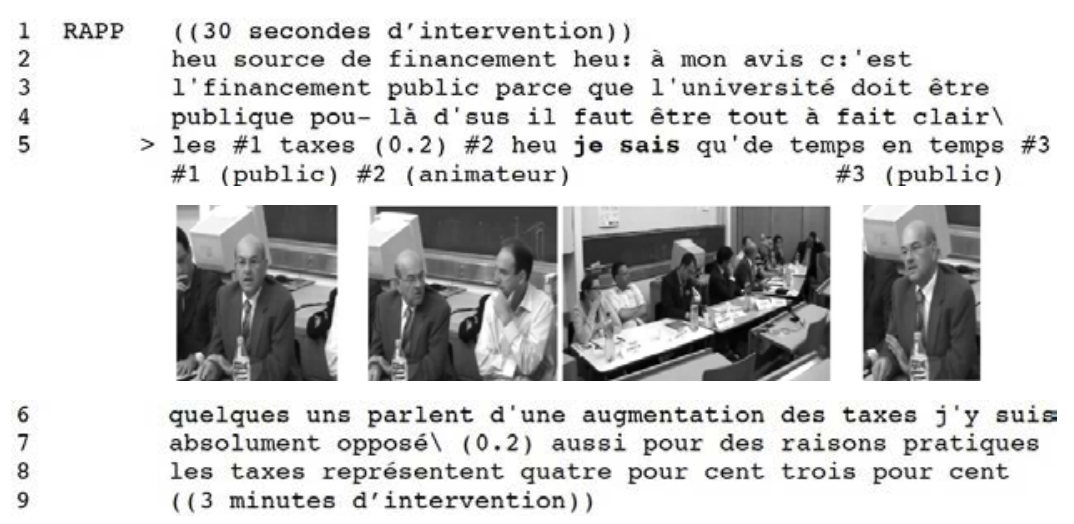

Au niveau multimodal, on constate un changement ponctuel de direction du regard, du public (images 1 et 3) vers l'animateur (image 2). Cette orientation vers l'animateur n'est pas sans lien avec la dimension dialogique de ce JE SAIS: la position prise en compte dans la complétive de JE SAIS relève d'une information d'arrière-plan - un ouï-dire - qui sollicite, à la manière de l'animateur, l'opposition contre-argumentée (voir aussi supra $\$ 3.2 .1$ ).

La séquence suivante est tirée de l'ouverture d'un débat télévisé consacré à l'initiative soutenue par Philippe Nantermod (NANT) et visant à limiter le droit de recours des associations écologistes en Suisse.

(5) RTS.REC-ECO / 00:00:00.000
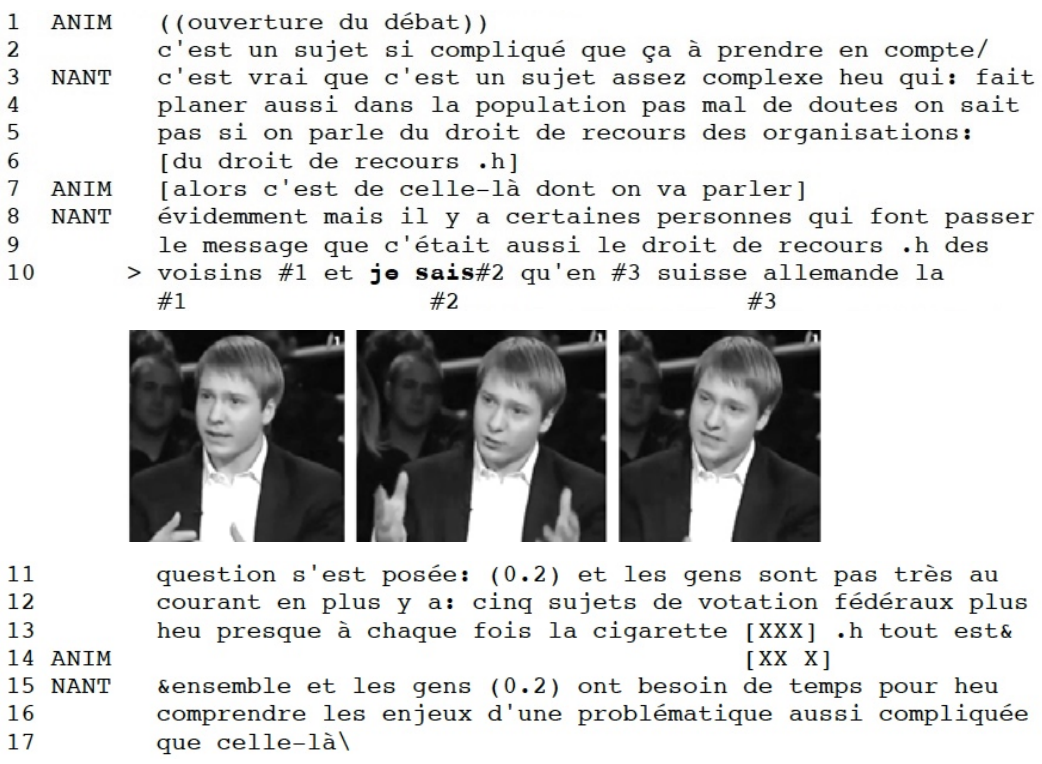

À la ligne 2, l'animateur conclut son introduction en interpelant l'invité sur la complexité du sujet abordé. La réponse de NANT aux lignes 3-6 est interrompue par le recadrage thématique 
demandé par l'animateur en ligne 7, qui entérine le sujet sur lequel il faut se concentrer ('c'est de celle-là dont on va parler') et cherche ainsi à bloquer la mention d'autres candidats. Le JE SAIS de la ligne 10 sert dès lors à NANT à légitimer, rétrospectivement, la trajectoire donnée à son tour. La légitimation se base sur la mention, en tant qu'objet de la complétive de JE SAIS, de la source énonciative du candidat 'le droit de recours des voisins' : cela a été évoqué en suisse allemande. Il s'agit là, davantage que dans les cas précédents, d'une information nouvelle dont on peut paraphraser l'introduction par ' $\mathrm{j}$ 'ai appris que...', et moins d'une information supposément déjà connue ('je sais bien que') ${ }^{12}$. Au niveau mimo-gestuel, notons que la production du JE SAIS est accompagnée d'un geste rapide et saillant de livraison des deux mains paumes ouvertes (images 1-3; 'livre entreouvert' chez Calbris (op. cit. 93-99), qui convient bien à l'introduction d'une information sur la scène du débat.

\subsubsection{Expertise : assise institutionnelle du dire}

Dans les extraits (4), (5) et (5b), l'information nouvelle introduite par JE SAIS relevait du témoignage, qu'il s'agisse de rapporter des propos ou une expérience personnelle. Les cas considérés ici sont plus spectaculaires dans la mesure où JE SAIS participe alors de la revendication d'une position épistémique forte légitimée par une catégorisation socioprofessionnelle.

L'extrait (6) est tiré de la toute fin du débat télévisé consacré à l'initiative visant à interdire les véhicules jugés trop polluants et trop dangereux. Martine Rebetez (REBE) est invitée par l'animateur à prendre position, notamment par rapport aux propos tenus par un allié dans le débat, Vincent Rossi, qui a expliqué la tournure prise par la discussion par un conflit générationnel entre lui et Charles Poncet (PONC), l'opposant.

(6) RTS.VEH-POL / 00:57:57.000

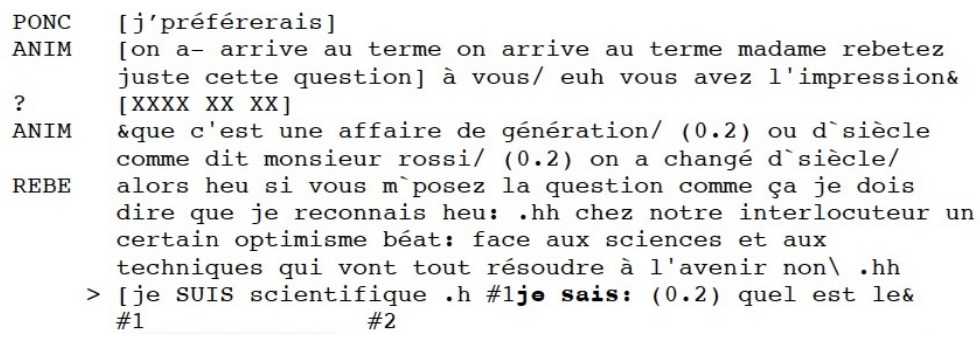

${ }^{12}$ De même, dans l'extrait (5b), tiré d'un débat public consacré à une initiative visant à introduire un salaire minimum en Suisse, le JE SAIS énoncé par un membre du public participe d'un témoignage personnel que le locuteur livre à ses allocutaires : ‘[... je me suis fait licencier mais j’ai pas été aux prud'hommes parce que à quelque part y a pas de conventions collectives, j'étais pas au courant de mon droit, j'y suis pas allé alors que je sais que j'aurais pu gagner, récupérer plutôt quatre mille cinq cents francs [...]'. 


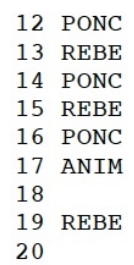

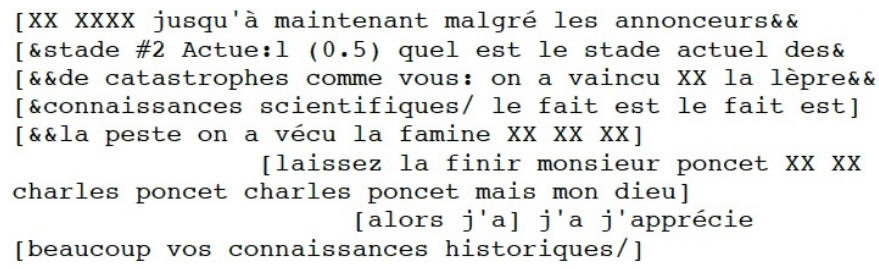

À la ligne 11, REBE s'auto-attribue une catégorie socio-professionnelle ('scientifique') avant d'introduire JE SAIS et sa complétive. Celle-ci contient des présents étendus et des syntagmes nominaux en première mention (par exemple 'le stade actuel des connaissances scientifiques'), qui donnent une certaine assise énonciative aux constats ainsi énoncés. L'auto-attribution d'un trait identitaire pertinent joue ici le rôle d'argument d'autorité, qu'on dit alors 'directe' dans la mesure où il n'est pas fait mention d'une voix externe mais l'autorité avancée est celle de la locutrice ou du locuteur (Doury 1998 ; Jacquin 2014, 338346).

Se présenter comme sachant relève ici d'une prise de position épistémique de l'ordre de l'expertise ${ }^{13}$. Et on constate que l'argument n'est pas subordonné à d'autres : il n'est pas glissé, comme incise, mais il est véritablement l'objet de l'argumentation à ce moment du discours et de l'interaction directe et cette fois très dynamique entre les participants, comme en témoignent les nombreux chevauchements. Au niveau multimodal, l'expression discursive de l'expertise se double d'une gestualité métaphorique de préhension sous la forme d'une pince angulaire plate (image 1) puis circulaire (image 2), dont la valeur de maitrise convient bien à l'effet de prise de position épistémique (Calbris op. cit.; Jacquin 2017b). À la différence de l'extrait (5), l'information introduite est en effet moins 'livrée' que 'saisie'.

\section{Conclusion}

La contribution s'est donné pour objectif d'examiner le marqueur épistémique emblématique JE SAIS dans un corpus francophone de débats publics et télévisés. Les 11 occurrences identifiées ont pour point commun de constituer des introducteurs de propositions complétives ('je sais $p$ ') émergeant en milieu de tours multi-UTC et non dans ses frontières. Il en va ainsi différemment des expressions épistémiques autonomes très fréquemment utilisées en tant que stratégies d'entrée ou de sortie de tour (par exemple 'I think' chez Kärkkäinen (2003) ; 'je sais pas' chez Pekarek Doehler (2016)). En d'autres termes, l'analysabilité de l'expression ne repose pas, ou du moins pas exclusivement, sur des mécanismes de coorientation tels qu'ils se manifestent dans la dynamique négociative. Les JE SAIS prennent sens dans des environnements discursifs certes dialogiques (présence de négations polyphoniques, de discours rapportés), mais relativement autonomes de l'organisation séquentielle, ce qui justifie donc d'en scruter les contextes énonciatifs et argumentatifs d'apparition et d'inviter la linguistique interactionnelle à une telle prise en compte.

Après avoir présenté les caractéristiques interactionnelles, argumentatives et énonciatives des débats telles que dégagées dans la littérature existante et telles qu'elles permettent de contextualiser l'émergence des JE SAIS, j'ai introduit différents facteurs pouvant expliquer

\footnotetext{
${ }^{13}$ Il en va de même dans l'extrait (6b), tiré du débat télévisé consacré au droit de recours des associations écologistes en Suisse et où Yves Nidegger (NIDE) condamne le chantage juridique opéré par les associations: '[...] on vend le temps procédural, on entend pas parler des conditions. Souvent, étant avocat, je sais que quand on négocie le retrait d'un recours, y a une convention, qu'elle est secrète et qu'il y a toujours des avantages. De l'autre côté [...]'. L'auto-attribution de la catégorie 'avocat' vient en appui de celle du savoir énoncé dans la complétive de JE SAIS.
} 
a priori la relativement faible fréquence de l'expression dans le corpus: le caractère rhétoriquement problématique de s'auto-attribuer du mérite, la possibilité, offerte par la langue française, d'utiliser le pronom indéfini on pour opacifier, adoucir cette auto-attribution et finalement la potentielle complexité fonctionnelle de JE SAIS, dont il n'est pas certain qu'il relève systématiquement et littéralement de la revendication d'un état de connaissance.

L'examen détaillé de la collection a fait apparaitre, derrière son apparente simplicité sémantique, cette polyfonctionnalité de la ressource JE SAIS dans le corpus. Comme on l'a rappelé supra, affirmer 'je sais $p$ ' constitue une manière de présenter $p$ comme présupposé. Ce faisant, il s'agit non seulement d'un moyen d'afficher, dans une certaine mesure, son savoir sur $p$, mais aussi d'une ressource permettant de sortir $p$ de la zone de confrontation. Les extraits analysés tendent à montrer que pour ne pas heurter certains principes rhétoriques, il faut toutefois que ces JE SAIS introduisent des éléments qui s'y prêtent, notamment des savoirs plus ou moins partagés a priori dont le caractère présupposé ne pose pas problème ou des éléments concédés à l'adversaire, ou alors qu'ils introduisent au contraire des arguments nouveaux, cette fois de manière étayée et donc relativement spectaculaire (par exemple "je suis scientifique, je sais $p^{\prime}$ ). On peut également relever la richesse multimodale qui caractérise l'énonciation des JE SAIS considérés ici. Dit grossièrement, il 'se passe' toujours quelque chose au niveau mimogestuel, qu'il s'agisse d'un changement ponctuel de direction du regard et/ou d'un geste saillant. Outre le fait qu'elles contribuent au balisage des énoncés contenant JE SAIS, ces actions s'articulent à leur orientation argumentative. C'est particulièrement visible au niveau des gestes de prise en compte de l'allocutaire dans l'extrait (1), d'intégration d'un élément concédé dans l'extrait (3), de livraison d'une information lors d'un témoignage dans l'extrait (4) ou encore de préhension experte d'un état de fait dans l'extrait (6).

Le parcours esquissé dans la présente contribution constitue une première tentative de rendre compte des positionnements épistémiques tels qu'ils participent à l'organisation de différentes activités argumentatives, dont les débats. En ce sens, elle est destinée à être complétée par une étude systématique d'autres expressions dans des contextes identiques ou différents.

Jérôme Jacquin

Université de Lausanne / Sciences du langage et de l'information

Anthropole-3134, 1015 Lausanne, Suisse

+41216922987

<jerome.jacquin@unil.ch>

Remerciements. La présente publication est soutenue par le Fonds National Suisse de la recherche [PZ00P1_168033]. L'auteur tient également à remercier les responsables du numéro ainsi que deux reviewers anonymes pour leur relecture attentive et leurs commentaires précieux.

Conventions de transcription. La parole est transcrite selon les conventions ICOR et la mimo-gestualité selon les conventions de L. Mondada :

<http://icar.univ-lyon2.fr/projets/corinte/documents/2013_Conv_ICOR_250313.pdf>et

<https://franz.unibas.ch/fileadmin/franz/user_upload/redaktion/Mondada_conv_multimodality.pdf >.

\section{Références}

Aristote (1991). Rhétorique. Paris, Gallimard.

Atkinson, J.M. (1984). Public Speaking and Audience Responses: Some Techniques for Inviting Applause. In Atkinson, J.M. \& Heritage, J. (eds), Structures of Social Action: Studies in Conversation Analysis, Cambridge/Paris, Cambridge University Press/Maison des Sciences de l'Homme, 370-409. 
Bovet, A. \& Malbois, F. (2011). (En)Jeux du cadre de participation dans la discussion publique médiatisée. In Burger, M., Jacquin, J. \& Micheli, R. (éds), La parole politique en confrontation dans les médias, Bruxelles, De Boeck Université, 51-68.

Burger, M. (2005). La complexité argumentative d'une séquence de débat politique médiatique. In Burger M. \& Martel, G. (éds), Argumentation et communication dans les médias, Québec, Nota Bene, 51-79.

Calbris, G. (2003). L'expression gestuelle de la pensée d'un homme politique. Paris, CNRS Editions.

Clayman, S.E. (1993). Booing: The Anatomy of a Disaffiliative Response. American Sociological Review, 58 (1), 110-130.

Clayman, S.E. \& Heritage, J. (2002). The News Interview: Journalists and Public Figures on the Air. Cambridge, Cambridge University Press.

Doury, M. (1997). Le débat immobile. L'argumentation dans le débat médiatique sur les parasciences. Paris, Kimé.

Doury, M. (1998). El argumento de autoridad en situación: el caso del debate mediático sobre astrología. Escritos-Revista del Centro de Ciencias del Lenguaje, 17/18, 89-112.

Doury, M. \& Kerbrat-Orecchioni, C. (2011). La place de l'accord dans l'argumentation polémique : le cas du débat Sarkozy/Royal (2007). A contrario, 16, 63-87.

Drew, P. \& Heritage, J. (eds) (1992). Talk at Work: Interaction in institutional settings. Cambridge, Cambridge University Press.

Edwards, D. \& Potter, J. (1992). Discursive Psychology. London, Sage Publications.

Gosselin, L. (2014). Sémantique des jugements épistémiques: degré de croyance et prise en charge. Langages, 193, 63-81.

Greatbatch, D. (1992). On the management of disagreement between news interviewees. In Drew, P. \& Heritage, J. (eds), Talk at Work: Interaction in institutional settings, Cambridge, Cambridge University Press, 268-301.

Heritage, J. \& Greatbatch, D. (1986). Generating Applause: A Study of Rhetoric and Response at Party Political Conferences. American Journal of Sociology, 92 (1), 110-157.

Heritage, J. \& Raymond, G. (2005). The Terms of Agreement: Indexing Epistemic Authority and Subordination in Talk-in-Interaction. Social Psychology Quarterly, 68 (1), 15-38.

Hutchby, I. (1996). Confrontation Talk: arguments, asymmetries, and power on talk radio. Mahwah, NJ, Lawrence Erlbaum Associates.

Hutchby, I. (1999). Rhetorical Strategies in Audience Participation Debates on Radio and TV. Research on Language and Social Interaction, 32(3), 243-267.

Hutchby, I. (2001). 'Witnessing': The use of first-hand knowledge in legitmating lay opinions on talk radio. Discourse Studies, 3 (4), 481-497.

Jackson, S. \& Jacobs, S. (1982). The collaborative production of proposals in conversational argument and persuasion. A study of disagreement regulation. Journal of the American Forensic Association, (18), 77-90.

Jacobs, S. \& Jackson, S. (1980). Structure of conversational argument: pragmatic bases for the enthymeme. The Quarterly Journal of Speech, 66, 251-265.

Jacobs, S. \& Jackson, S. (1982). Conversational argument : A discourse analytic approach. In Cox, R.J. \& Willard, C.A. (eds), Advances in Argumentation Theory and Research. Carbondale and Edwardsville, Southern Illinois University Press, 205-237.

Jacobs, S. \& Jackson, S. (1989). Building a model of conversational argument. In Dervin, B., Grossberg, L, O'Keefe, B.J \& al. (eds), Rethinking communication, Newbury Park, Sage, 153-171.

Jacquin, J. (2014). Débattre. L'argumentation et l'identité au cœur d'une pratique verbale. Bruxelles, De Boeck.

Jacquin, J. (2017a). Le pronom ON dans l'interaction en face à face: Une ressource de (dé)contextualisation. Langue française, 193 (1), 77-92.

Jacquin, J. (2017b). 'Prenons par exemple...'. Gestes métaphoriques de préhension dans l'argumentation en co-présence. In Bonhomme, M., Paillet, A.M. \& Wahl, P. (éds), Métaphore et argumentation, Louvain-la-Neuve, Academia-L'Harmattan, 221-239. 
Jacquin, J. \& Gradoux, X. (éds) (2015). L'organisation de l'interaction au niveau d'analyse intermédiaire/ The Organization of Interaction at the Intermediate Level of Analysis. Bulletin Suisse de Linguistique Appliquée, 101.

Jacquin, J. \& Micheli, R. (2012). Entre texte et interaction: propositions méthodologiques pour une approche discursive de l'argumentation en sciences du langage. In Neveu, F., Muni Toke, V., Blumenthal, P. \& al. (éds), Actes du $3^{e}$ Congrès Mondial de Linguistique Française, Lyon, EDP Sciences, 599-611.

Jacquin, J. \& Micheli, R. (2013). Dire et montrer qui on est et ce que l'on ressent : une étude des modes de sémiotisation de l'identité et de l'émotion. In Constantin de Chanay, H., Colas-Blaise, M. \& Le Guern, O. (éds), Dire / Montrer. Au cœur du sens, Chambéry, Université de Savoie, 67-92.

Jonasson, K. (2006). Le pronom clitique ON : un outil de non-partition référentielle. In Kleiber, G., Schnedecker, C. \& Theissen, A. (éds), La relation Partie-Tout, Louvain, Peeters, 59-72.

Kärkkäinen, E. (2003). Epistemic Stance in English Conversation. Amsterdam, Benjamins.

Kotthoff, H. (1993). Disagreement and Concession in Disputes: On the Context Sensitivity of Preference Structures. Language in Society, 22 (2), 193-216.

Lamy, B. (1741). La Rhétorique ou l'Art de parler. Paris, François Mathey.

Livingstone, S. \& Lunt, P. (1994). Talk on television: audience participation and public debate. London/New York, Routledge.

Llewellyn, N. (2005). Audience Participation in Political Discourse: A Study of Public Meetings. Sociology, 39 (4), 697-716.

McIlvenny, P. (1996). Heckling in Hyde Park: Verbal Audience Participation in Popular Public Discourse. Language in Society, 25 (1), 27-60.

Mondada, L. (2013). Embodied and spatial resources for turn-taking in institutional multi-party interactions: Participatory democracy debates. Journal of Pragmatics, 46 (1), 39-68.

Pekarek Doehler, S. (2016). More than an epistemic hedge: French 'je sais pas' ('I don't know') as a resource for the sequential organization of turns and actions. Journal of Pragmatics, 106, 148-162.

Plantin, C. (1996). Le trilogue argumentatif. Présentation de modèle, analyse de cas. Langue française, (112), 9-30.

Plantin, C. (2005). L'argumentation : histoire, théories et perspectives. Paris, PUF.

Pomerantz, A. (1984). Agreeing and disagreeing with assessments: some features of preferred/dispreferred turn shapes. In Atkinson, J.M. \& Heritage, J. (eds), Structures of Social Action: Studies in Conversation Analysis, Cambridge/Paris, Cambridge University Press/Maisons des Sciences de l'Homme, 57-101.

Rabatel, A. (2001). La valeur de 'on' pronom indéfini/pronom personnel dans les perceptions représentées. L'information grammaticale, 88, 28-32.

Raymond, G. \& Heritage, J. (2006). The Epistemics of Social Relations: Owning Grandchildren. Language in Society, 35(05), 677-705.

Relieu, M. \& Brock, F. (1995). L'infrastructure conversationnelle de la parole publique: L'analyse des réunions politiques et des interviews télédiffusées. Politix, 31, 77-112.

Sacks, H., Schegloff, E.A. \& Jefferson, G. (1974). A Simplest Systematics for the Organization of TurnTaking for Conversation. Language, 50(4), 696-735.

Selting, M. (2000). The construction of units in conversational talk. Language in Society, 29 (4), 477-517.

Thornborrow, J. (1997). Having their say: The function of stories in talk-show discourse. Text, 17 (2), 241-262.

Van Eemeren, F.H., \& Grootendorst, R. (2004). A Systematic Theory of Argumentation: the pragmadialectical approach. Cambridge, Cambridge University Press.

Vet, C. (1994). Savoir et croire. Langue française, 102 (1), 56-68. 